\title{
DETECTION OF METALLO BETA LACTAMASE PRODUCTION IN IMIPENEM RESISTANT GRAM NEGATIVE BACILLI NON FERMENTERS ISOLATED IN A TERTIARY CARE HOSPITAL.
}

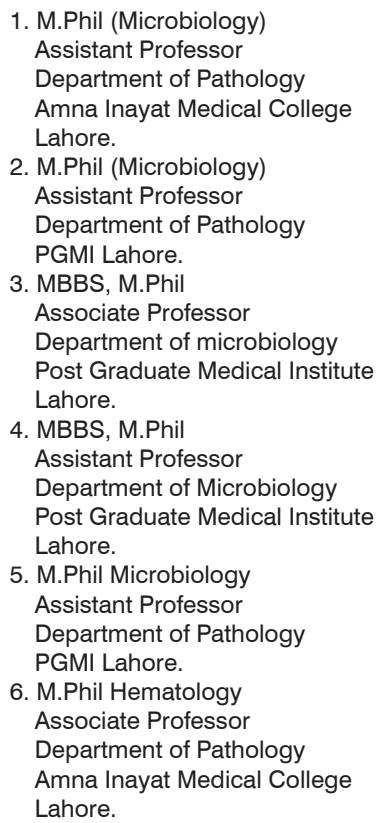

2. M.Phil (Microbiology)

Assistant Professor

Department of Pathology

PGMI Lahore.

3. MBBS, M.Phil

Associate Professor

Department of microbiology

Post Graduate Medical Institute Lahore.

4. MBBS, M.Phil

Assistant Professor

Department of Microbiology

Post Graduate Medical Institute

Lahore.

5. M.Phil Microbiology

Assistant Professor

Department of Pathology

PGMI Lahore.

6. M.Phil Hematology

Associate Professor

Department of Pathology

Amna Inayat Medical College

Lahore.

Correspondence Address:

Dr. Muna Malik

Department of Pathology

PGMI, Lahore Pakistan.

mmkamboh@hotmail.com

Article received on:

09/01/2019

Accepted for publication:

10/04/2019

\begin{abstract}
Ayesha Sajjad1, Muna Malik², Iffat Javed ${ }^{3}$, Sohaila Mushtaq ${ }^{4}$, Fareeha Imran ${ }^{5}$, Rabiya Jamil $^{6}$
\end{abstract}
\begin{abstract}
Objectives: Metallo-beta lactamase (MBL) producing non fermenter Gram negative bacilli is an emerging warning and cause of worry as they have established as one of the most feared resistance mechanisms and are the foremost cause of nosocomial infections worldwide. Carbapenem, including Imipenem, Meropenem and Doripenem are often used as a last remedy for treatment of infections caused by Pseudomonas aeruginosa, Acinetobacter and other Gram-negative. The present study was designed to explore the distribution of imipenem resistant non-fermenter Gram-negative bacilli isolates in different age groups. Study Design: Cross sectional Descriptive study. Setting: Microbiology laboratory, PGMI, Lahore. Period: January 2015 to December 2015. Material \& Methods: 53 imipenem resistant NFGNB that were isolated from appropriate sampling of patients suffering from several infections were analyzed by using different standard microbiological techniques like microscopy, culture methods, biochemical reactions and antibiotic susceptibility using Kirby-Bauer method. MBL recognition was performed by imipenem-2MPA double disc synergy test (DDST). Results: This study shows the frequency of imipenem resistant non-fermenter Gram-negative bacilli isolated from various clinical wards. Maximum NFGNB were recovered from surgery/surgical allied $35.84 \%$ followed by ICU $28.3 \%$, medicine /medicine allied $20.75 \%$, pediatrics $9.4 \%$ and gynae/ obs $5.6 \%$ respectively. MBL production was identified among different imipenem resistant non-fermenter Gram-negative bacilli isolates by DDST using 2-MPA. Out of total 53 imipenem resistant non-fermenter Gram Negative Bacilli 37 Pseudomonas aeruginosa 20(54.05\%) were MBL positive. Out of 13 Acinetobacter baumannii and 2 Pseudomonas luteola, 11(84.61\%) Acinetobacter baumannii and 1(50\%) Pseudomonas luteola were positive for MBL production. None of the Acinetobacter junii indicated MBL production. Conclusion: Double disc synergy test is operational for detection of MBL producers among NFGNB. It can be established in our routine clinical microbiology laboratories, for the MBL recognition especially in imipenem resistant isolates as of its cost efficiency.
\end{abstract}

Key words: $\quad$ Carbapenems, Double Disc Synergy Test, Metallo-Beta-Lactamase, Mercaptopropionic Acid, Non-Fermenting Gram-Negative Bacilli.

Article Citation: Sajjad A, Malik M, Javed I, Mushtaq S, Imran F, Jamil R. Detection of metallo beta lactamase production in imipenem resistant gram negative bacilli non fermenters isolated in a Tertiary Care Hospital. Professional Med J 2019; 26(12):2080-2084. DOI: 10.29309/TPMJ/2019.26.12.3073

\section{INTRODUCTION}

Metallo beta lactamase (MBL) producing nonfermenter Gram negative bacilli is an evolving intimidation and cause of terror, as they have established as one of the most dreaded resistance mechanisms and are the prominent cause of nosocomial infections globally. ${ }^{1}$ Carbapenem, including imipenem, meropenem and doripenem are often used as a last option for treatment of infections, which caused by Pseudomonas aeruginosa, Acinetobacter and other Gram-negative bacteria. However, carbapenem-resistant $P$. aeruginosa has become prevalent worldwide. ${ }^{2}$ Carbapenem resistance is mainly due to up regulation of efflux pumps, reduced outer membrane permeability. ${ }^{3}$ MBLs are reticent by metal chelators, such as an ethylenediamine tetra acetic acid (EDTA) and thiol-based compounds. MBL-encoding genes have been testified worldwide in GNB, such as Pseudomonas spp. Acinetobacter spp, and members of the Enterobacteriaceae family. ${ }^{4}$ The non-fermenter Pseudomonas aeruginosa is a well-known dreaded pathogen in hospital surroundings and 
play a noteworthy role in causing nosocomial outbreaks among susceptible patients, especially mechanically ventilated patients VAP. ${ }^{5}$ It is estimated that at least 2 million people gain bacterial infections that are resistant to standard therapy each year only in in the United States. ${ }^{6}$

Up to $25 \%$ of cutaneous colonization is caused by Acinetobacter and are the most common bacteria found on the skin of hospital personnel, which, often a times, cause a number of outbreaks of nosocomial infections such as septicemia, pneumonia, wound sepsis, endocarditis, meningitis, urinary tract infections. ${ }^{7}$ Eventually, infection control specialists and clinical microbiologists need to work together to regulate the risk carried by Carbapenem resistant non fermenting Gram-negative bacilli in their institution and what methods should be measured for surveillance and revealing of these pathogens.

\section{METHODOLOGY}

This descriptive study has been conducted in the Microbiology department of Post Graduate Medical Institute from January 2015 to December 2015. All the labeled samples received in microbiology laboratory from Lahore General Hospital were inoculated on Blood agar and MacConkey agar and incubated at $37^{\circ} \mathrm{C}$ for 24 hours. Non-lactose fermentation was noted on Mac Conkey agar. Preliminary identification of NFGNB was done by Gram staining, Catalase and Oxidase test. The final confirmation of all the NFGNB was done upto specie level by Analytical profile index Non Enterobactericae Kit (API $20 \mathrm{NE}$ Biomerieux). Phenotypic verification of provisional MBL producers was done by Double Disc Synergy Test. Isolates which were imipenem resistant were advance investigated for Metallobeta lactamase production by double disc synergy method. The bacterial suspension with turbidity equivalent to $0.5 \mathrm{Mc}$ Farland standards was arranged and inoculated on Mueller Hinton agar.

After inoculation of the experimental organism on Muller Hinton agar, 2 imipenem disc containing 10 micrograms were positioned on the surface of agar plate at the distance of $4-5 \mathrm{~cm}$ center to center. A disc comprising 3 microliters of diluted 1:8 2-Mercaptopropionic acid was placed near one of the IPM disc at a distance of $1-1.5 \mathrm{~cm}$. The plates were incubated at $37^{\circ} \mathrm{C}$ overnight. The expansion of the diameter of growth inhibitory zone around imipenem and 1:8 2-Mercaptopropionic containing disc by $\geq 7 \mathrm{~mm}$ was reflected as positive for MBL production. Quality control of Pseudomonas aeruginosa (ATCC 27853) and Stenotrophomonas maltophilia (ATCC 13636) was run with each batch of the test. The results were interpreted as per Clinical and Laboratory Standard Institute guidelines.

\section{RESULTS}

Total 53 imipenem resistant non-fermenter Gramnegative bacilli were isolated for culture and sensitivity during the study period. Positive synergy test is shown in Figure-1. The imipenem resistant NFGNB isolates was distributed according to the age of the patients shown in Figure-2. Table-I. Shows frequency of imipenem resistant non-fermenter Gram-negative bacilli isolates recovered from several clinical wards. Among 53 imipenem resistant non-fermenter Gram-negative bacilli, most common non-fermenter bacteria isolated was Pseudomonas aeroginosa 37 $(69.81 \%)$ rest of the results showed in Table-II. In our study, most common MBL producing bacteria was Pseudomonas aeruginosa 20 (54.05\%), rest of MBL making bacteria are shown in Table-III.

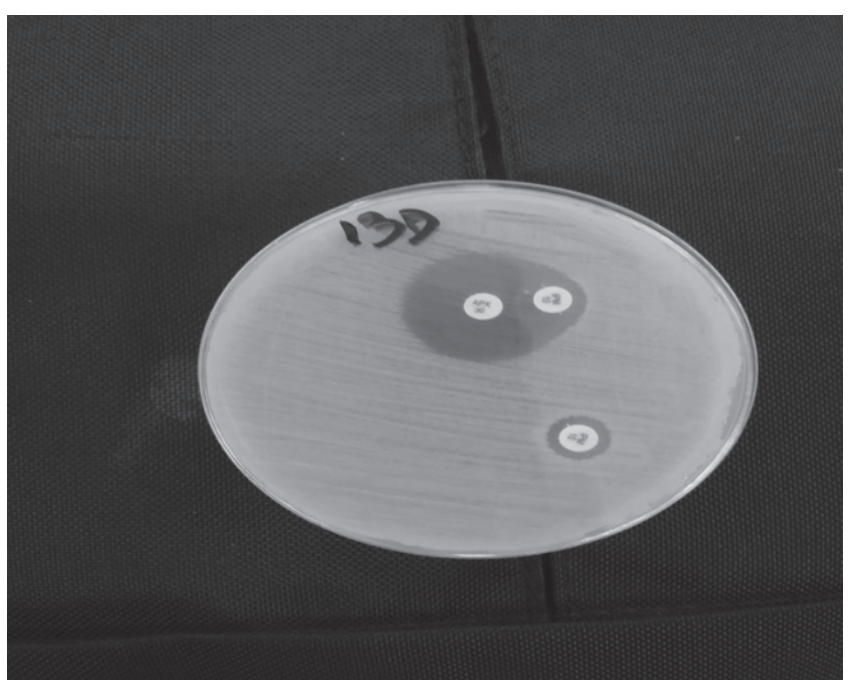

Figure-1. Double disc synergy test showing positive synergy test. 


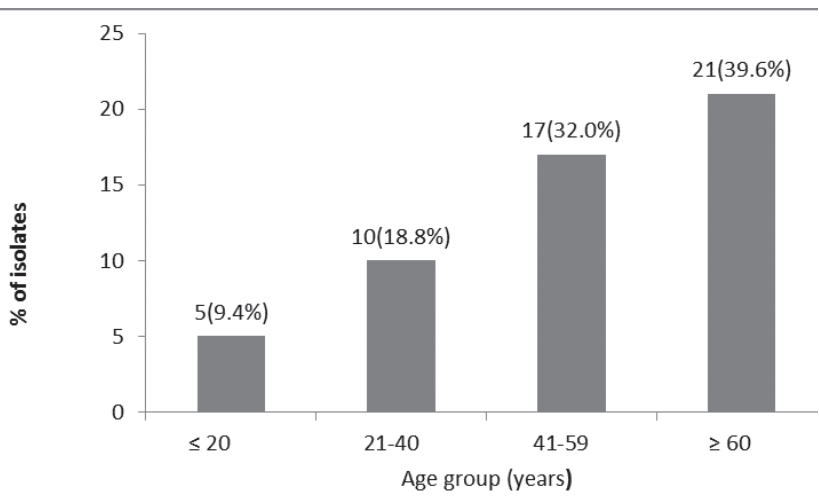

Figure-2. The distribution of imipenem resistant nonfermenter Gram negative bacilli isolates according to age $(n=53)$

\begin{tabular}{|c|c|c|c|}
\hline Ward & \multicolumn{2}{|c|}{$\begin{array}{c}\text { No. of } \\
\text { Isolates }\end{array}$} & $\begin{array}{c}\text { Percentage } \\
(\%)\end{array}$ \\
\hline Medicine/Medicine Allied & \multicolumn{2}{|r|}{11} & $20.75 \%$ \\
\hline Surgery/Surgical Allied & \multicolumn{2}{|r|}{19} & $35.84 \%$ \\
\hline Gynae/Obs & \multicolumn{2}{|r|}{3} & $5.66 \%$ \\
\hline ICU & \multicolumn{2}{|r|}{15} & $28.3 \%$ \\
\hline Pediatrics & \multicolumn{2}{|r|}{5} & $9.4 \%$ \\
\hline \multicolumn{4}{|c|}{$\begin{array}{l}\text { Table-I. Frequency of imipenem resistant non } \\
\text { fermenter Gram negative bacilli isolated from several } \\
\text { clinical wards }(n=53)\end{array}$} \\
\hline Species & \multicolumn{2}{|c|}{$\begin{array}{c}\text { No of } \\
\text { Isolates }\end{array}$} & $\begin{array}{c}\text { Percentage } \\
(\%)\end{array}$ \\
\hline Pseudomonas Aeruginosa & \multicolumn{2}{|r|}{37} & $69.81 \%$ \\
\hline Acinetobacter Bauminii & \multicolumn{2}{|r|}{13} & $24.52 \%$ \\
\hline Pseudomonas Luteola & \multicolumn{2}{|r|}{2} & $3.77 \%$ \\
\hline Acinetobacter Junii & \multicolumn{2}{|r|}{1} & $1.88 \%$ \\
\hline \multicolumn{4}{|c|}{$\begin{array}{l}\text { Table-II. Frequency of different Imipenem resistant } \\
\text { non-fermenter Gram negative bacilli isolates in } \\
\text { different clinical specimens }(n=53)\end{array}$} \\
\hline NFGNB isolated $(n=53)$ & & $\begin{array}{l}\text { MBL } \\
\text { Positive } \\
\text { on DDST }\end{array}$ & $\begin{array}{l}\text { Per- } \\
\text { centage } \\
(\%)\end{array}$ \\
\hline \multicolumn{2}{|c|}{ Pseudomonas Aeruginosa (37) } & 20 & $54.05 \%$ \\
\hline \multicolumn{2}{|c|}{ Acinetobacter Baumannii (13) } & 11 & $84.61 \%$ \\
\hline \multicolumn{2}{|l|}{ Pseudomonas Luteola (2) } & 1 & $50 \%$ \\
\hline Acinetobacter Junii (1) & & 0 & $0 \%$ \\
\hline \multicolumn{4}{|c|}{$\begin{array}{l}\text { Table-III. Frequency of Metallo beta lactamase } \\
\text { creating different non fermenter Gram negative bacilli } \\
\text { isolates by double disc synergy test using 2-MPA } \\
\text { disk }(n=53)\end{array}$} \\
\hline
\end{tabular}

Professional Med J 2019;26(12):2080-2084.

\section{DISCUSSION}

Due to excessive and irrational use of carbapenems, a shocking increase in carbapenem resistance has been recounted in many Gramnegative bacteria including Acinetobacter spp. ${ }^{8}$ The resistance produced by broad-spectrum cephalosporin is a tenacious problem in managing infections caused by Pseudomonas areuginosa, and Enterobacter species, as well as other Enterobacteriaceae. ${ }^{9}$ There are limited treatment options for carbapenem - resistant Acinetobacter baumannii and Enterobacteriaceae, so clinicians must completely evaluate all offered therapeutic options for treating these multidrug-resistant organisms. ${ }^{10}$ The present study showed the dispersal of imipenem resistant non-fermenter Gram-negative bacilli isolates according to age. The maximum age in which we stated distribution of imipenem resistance was $39.6 \%$ in 60 years. Similarly, a study was conducted in hospitals of Kermansha by Akya et al in 2015, which conveyed $36.4 \%$ imipenem resistant isolates in age group of 60 years and above. ${ }^{11}$ Another study conducted by Lefevre et al in 2013 reported maximum isolation of NFGNB between 50-60 years of age. ${ }^{12}$ In contrast, El-Mahallawy et al in 2015 in Egypt reported the high prevalence in age group of 48 years. ${ }^{13}$

Table-I of our study shows the frequency of imipenem resistant non-fermenter Gram-negative bacilli isolates recovered from several clinical wards. Maximum NFGNB were recovered from surgery/surgical allied $35.84 \%$. The results were nearly similar to the study conducted by Prasanna et al in 2016. They reported maximum imipenem resistant NFGNB recovered from surgical wards $30 \%$ followed by surgical ICU $20.5 \% .{ }^{14}$ One more study conducted by Maniyan et al in 2016 indicated high prevalence of non-fermenters from surgical wards $40 \%$ followed by ICU $20 \% .^{15}$ Contrary to this, some of the researches however stated maximum recovery of NFGNB from intensive care units. Dash et al in 2013 in India reported utmost number of isolates recovered from ICU $45.2 \%$. $^{16}$ Table-Il of our study shows the frequency of imipenem resistant non-fermenter Gram-negative bacilli isolates recovered from different clinical specimen. Amongst 53 imipenem resistant non- 
fermenter Gram-negative bacilli 37 (69.81\%) were Pseudomonas aeroginosa. Our results were alike with the number of studies such as study carried out chawla et al in 2013; they recounted that Pseudomonas aeruginosa was the most commonly isolated non-fermenter pathogen. ${ }^{17}$ In difference to our study, Goel et al in 2013 in tertiary care hospital of India described that Acinetobacter baumannii $48.78 \%$ was the chief isolated pathogen among the non-fermenters trailed by Pseudomonas aeruginosa $31.71 \%{ }^{18}$

Table-III shows percentage of imipenem nonsusceptible metallo-beta lactamase producing different non-fermenter Gram-negative bacilli isolates by Double disc synergy test. All 53 imipenem resistant NFGNB were tested for MBL production by DDST using imipenem disc only and in combination with 2-MPA. Out of 53 imipenem resistant, NFGNB isolates 20 (54.05\%) Pseudomonas aeruginosa, 11(84.61\%) Acinetobacter baumannii, 1(50\%) Pseudomonas luteola were originate positive for MBL production by DDST test. Acinetobacter junii did not show MBL production on DDST. We establish high percentage of MBL production in Acinetobacter baumannii in this study, which is in harmony to the study conducted by Kabbaj et al in 2013 in Morocco, which revealed $74 \%$ of MBL producing Acinetobacter baumannii. Among imipenem resistant isolates which is parallel to our study. ${ }^{19}$ In contrast to our study, Ahir et al in 2012 mentioned the prevalence of MBL in P.aeroginosa to be as high as $11.42 \%$ followed by $10.40 \%$ in Acinetobacter spp. Tellis et al in 2013 in India also stated the high percentage of MBL producing Pseudomonas aeruginosa. ${ }^{20,21}$

\section{CONCLUSION}

Non-fermenting Gram-negative bacilli isolated from diverse clinical specimens should not be overlooked and recognized by using standard methods, to institute appropriate and timely antibiotic reporting. There is an alarmingly high rate of resistance to many antimicrobials created by the non-fermenter Gram-negative bacilli including carbapenems. We suggest that further studies should be carried out to assess the usefulness of older and newer antimicrobial agents to prevent the emergence of multi drug resistant bacteria in clinical spacimens.

\section{Copyright@ 10 Apr, 2019.}

\section{REFERENCES}

1. Khan AU, Maryam L and Zarrilli R. Genetics and worldwide spread of New Delhi Metallo- $\beta$-lactamase (NDM): A threat to public health. BMC Microbiol 2017; $17: 101$.

2. Acharya M, Joshi PR, Thapa K, Aryal R, Kakshapati T and Sharma $S$. Detection of metallo- $\beta$-lactamasesencoding genes among clinical isolates of pseudomonas aeruginosa in a Tertiary Care Hospital, Kathmandu, Nepal. BMC Res Notes 2017; 10: 718.

3. Meletis G. Carbapenem resistance: Overview of the problem and future perspectives. The Adv Infect Dis 2016; 3(1): 15-21.

4. Panchal CA, Oza SS, Mehta SJ. Comparison of four phenotypic methods for detection of metallo- $\beta$ lactamase-producing Gram-negative bacteria in rural teaching hospital. J Lab Physicians.2015; 9(2): 81-83.

5. Cornaglia G, Mazzariol A, Lauretti I, Rossolini GM, Fontana R. Hospital Outbreak of CarbapenemResistant Pseudomonas aeruginosa Producing VIM1, a Novel Transferable Metallo- $\beta$-Lactamase. Clin Infect Dis 2000; 31(5): 1119-25.

6. Harris $A D$, Smith $D$, Johnson JA, Bradham DD, Roghmann MC. Risk factors for imipenem-resistant pseudomonas aeruginosa among Hospitalized Patients. Clin Infect Dis 2002; 34 (3): 340-45.

7. Moradi J, Hashemi FB, Bahador A. Antibiotic resistance of acinetobacter baumannii in Iran: A systemic review of the published literature. Osong Public Health Res Perspect 2015; 6(2): 79-86.

8. Codjoe FS and Eric S. Donkor, ES. Carbapenem resistance: A Review Med Sci 2018; 6, 1.

9. Kaye KS. \& Kaye D. Multidrug-resistant pathogens: Mechanisms of resistance and epidemiology. Curr. Infect Dis Rep 2000; 2: 391.

10. Perez F, Hujer AM, Hujer K M, Decker BK, Rather $\mathrm{PN}$, Bonomo RA. Global challenge of multidrugresistant acinetobacter baumannii. Antimicrob Agents Chemother. 2007; 51(10): 3471-84.

11. Akya, A, Salimi A, Nomanpour B, Ahmadi K. Prevalence and clonal dissemination of metallo-beta-lactamaseproducing pseudomonas aeruginosa in Kermanshah. Jundishapur J Microbiol 2015; 8(7): e20980. 
12. Lefèvre LA, Angebault C, Barbier F, Hamelet E, Defrance G, Ruppé E, BronchardR, Lepeule R, Lucet JC, Mniai AE, Wolff M, Montravers P, Plésiat P, Andremont A. Emergence of imipenem-resistant gram-negative bacilli in intestinal flora of intensive care patients. Antimicrob Agents Chemother 2013; 57(3): 1488-95.

13. El-Mahallawy HA, Hassan SS, El-Wakil M, Moneer MM, Lobna Shalaby L. Increasing antimicrobial resistance monitored in surveillance analysis of blood stream infections in febrile neutropenic pediatric oncology patients. Asian Pac J Cancer Prev 2015; 16(14): 569195.

14. Prasanna S, Dharanidevi S, Das NK, Raj S. Prevalence, phenotypic characterization and antibiotic susceptibility of non-fermentative gram negative bacilli isolates at a Tertiary Care Centre. Int $\mathrm{J}$ Curr Microbiol App Sci. 2016; 5(11): 442-454.

15. Maniyan G, Vedachalam D, Chinnusamy N. Characterization and antimicrobial susceptibility pattern of non-fermenting gram negative bacilli from various clinical samples in a tertiary care hospital. Indian J Microbiol. Res 2016; 3(4):387-91.

16. Dash M, Padhi S, Pattnaik S, Mohanty I, Misra $P$. Frequency, risk factors, and antibiogram of Acinetobacter species isolated from various clinical samples in a tertiary care hospital in Odisha, India. Avicenna J Med 2013; 3(4): 97-102.
17. Chawla K, Vishwanath S, Frenil C Munim FC. Nonfermenting gram-negative Bacilli other than Pseudomonas aeruginosa and Acinetobacter Spp. Causing respiratory tract infections in a Tertiary Care Center. J Global Infect Dis 2013; 5(4):144-48.

18. Goel V, Hogade SA, S G Karadesai, SG. Prevalence of extended-spectrum beta-lactamases, AmpC betalactamase, and metallo-beta-lactamase producing Pseudomonas aeruginosa and Acinetobacter baumannii in an intensive care unit in a tertiary care hospital. J Scientific Socity 2013; 40(1):28-31.

19. Kabbaj H, Seffar M, Belefquih B, Akka D, Handor N, Amor $M$, Alaoui AE. Prevalence of Metallo- $\beta$-Lactamases Producing Acinetobacter baumannii in a moroccan Hospital. ISRN Infectious Diseases. 2012 Dec 3;2013.

20. Ahir HR, Patel PH, Berry RA, Parmar R, Soni ST, Shah PK, Vegad MM, Patil S. Prevelance of Metallo$\beta$-Lactamases producing pseudomonas and acinetobacter species in Tertiary Care Teaching Hospital, Gujrat. Int J Microbiol Res 2012; 4(9): 322-25.

21. Tellis R, S Muralidharan, Peter Al. Evaluation of three phenotypic methods for the detection of metallobetalactamase production in non-fermenting gram negative bacilli. Inter J Biomed Adv. Res. 2013; 04 (05): 300-5.

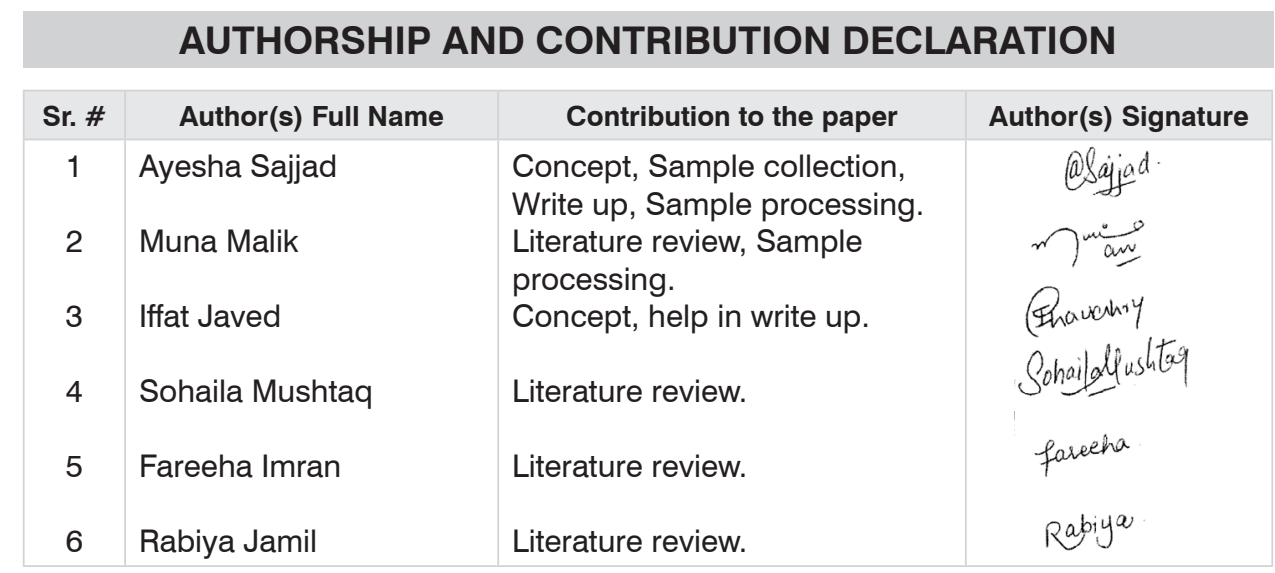

